\title{
Coplanar versus Non-coplanar Carboxyl groups: The Influence of Sterically Enforced Non-Coplanarity on the 2D Mixing Behavior of Benzene Tricarboxylic Acids
}

Robby Reynaerts, ${ }^{\dagger}$ Andrea Minoia, ${ }^{+}$Sai Manoj Gali, ${ }^{\dagger}$ Lakshya Daukiya, ${ }^{\dagger}$ Niels Van Velthoven,, Dirk De Vos, ${ }^{\#}$ Roberto Lazzaroni, ${ }^{* *}$ Kunal S. Mali ${ }^{\dagger *}$ and Steven De Feyter ${ }^{\dagger *}$

${ }^{\dagger}$ Department of Chemistry, Division of Molecular Imaging and Photonics, KU Leuven, Celestijnenlaan 200F, B-3001 Leuven, Belgium, *Laboratory for Chemistry of Novel Materials, Materials Research Institute, University of Mons, Place du Parc 20, 7000 Mons, Belgium, Centre for Surface Chemistry and Catalysis, Celestijnenlaan 200F, B-3001 Leuven, Belgium

\begin{abstract}
Corresponding authors: roberto.lazzaroni@umons.ac.be, kunal.mali@kuleuven.be, steven.defeyter@kuleuven.be
\end{abstract}

\begin{abstract}
:
Hydrogen bonding is an indispensable tenet in the fabrication of surface-confined physisorbed supramolecular networks. On-surface supramolecular chemistry is dominated by aromatic carboxylic acids which allow implementation of highly directional and robust design elements in the form of hydrogen bonds. In this contribution, we investigate the influence of sterically enforced non-coplanarity of the carboxyl groups on the hydrogen bonding ability and selfassembly behavior of iodinated benzene tricarboxylic acid at the solution/graphite interface. The carboxylic groups of this acid are non-coplanar with respect to the benzene ring because of the bulky iodine atoms substituted on the ring. The self-assembled networks formed at the solutionsolid interface were characterized at sub-molecular resolution using scanning tunneling microscopy (STM). The assembly behavior was scrutinized further by employing detailed molecular modelling simulations that provide an insight into the energetics of the self-assembled network formation. The on-surface mixing behavior of the iodinated, and the non-iodinated analogue, the widely studied trimesic acid, was investigated. STM reveals that deposition of the
\end{abstract}


two compounds on the graphite surface leads to phase separation orthogonal to the substrate and yields a supramolecular heterostructure with a well-defined bilayer. The present results indicate that, while the non-coplanarity induced by steric factors is not detrimental to the assembly behavior, it certainly contributes to the peculiar mixing behavior observed at the solutiongraphite interface. 


\section{Introduction}

Hydrogen bonding, with its all-pervasive reach, remains a highly reliable and widely exploited intermolecular interaction in supramolecular chemistry. Hydrogen bonds exhibit a number of desirable attributes such as co-operativity, directionality and selectivity. Their intermediate strength often provides a perfect balance between lability and stability allowing error correction via reversible bond formation leading to crystalline self-assembled networks. Carboxylic groups are arguably the most widely used synthons for hydrogen bonding. Each carboxyl group exhibits self-complementary hydrogen bonding ability where the oxygen atom of the $\mathrm{C}=\mathrm{O}$ group acts as a hydrogen-bond acceptor, whereas the $-\mathrm{OH}$ group acts as a hydrogen-bond donor. Thus, two carboxylic groups can form a cyclic dimer held together by two equivalent hydrogen bonds. Besides dimers, carboxylic groups can also be involved in trimeric and catemeric motifs. ${ }^{1,2}$

Supramolecular chemistry of carboxylic acids, in particular aromatic carboxylic acids, has been intensively studied in a diverse range of environments including the solid state, the solution phase and as thin films adsorbed on solid surfaces. On-surface supramolecular chemistry, where physisorbed monolayers are characterized using scanning probe microscopy, has rapidly gained popularity in the past couple of decades. Scanning tunneling microscopy (STM) allows direct visualization of supramolecular networks physisorbed on solid surfaces such as highly oriented pyrolytic graphite (HOPG) and $\mathrm{Au}(111)$ at sub-molecular resolution. ${ }^{3-5}$ Atomic force microscopy (AFM) has also been successfully applied for the visualization of hydrogen bonded monolayers on solid surfaces under ambient conditions. ${ }^{6,7}$

While functionalized alkanes and alkylated aromatic molecules were the most intensively studied systems in the early days of STM at the solution-solid interface, ${ }^{8}$ benzenecarboxylic acids served as an archetypal example of hydrogen bonded systems at a later stage. ${ }^{5}$ In order to form an extended self-assembled network, two appropriately placed carboxyl groups per molecule are necessary. The substitution pattern of these groups often determines whether or not a stable network is obtained, and also the geometry of the self-assembled network. Thus, despite 
possessing two carboxyl groups, phthalic acid (PA, benzene-1,2-dicarboxylic acid) does not form stable networks at the solution-solid interface whereas isophthalic acid (ISA, benzene-1,3dicarboxylic acid) and terephthalic acid (TA, benzene-1,4-dicarboxylic acid) do. ISA and TA both form self-assembled networks based on 1D hydrogen bonded arrays. The two network structures however are different and are determined by the substitution pattern: ISA forms zigzag whereas TA forms straight rows of hydrogen bonded dimers. ${ }^{9}$

The real interest in the on-surface self-assembly of aromatic carboxylic acids rose steeply with the STM characterization of the self-assembled networks of benzene-1,3,5-tricarboxylic acid (trimesic acid, TMA, Figure 1) under UHV conditions. ${ }^{10}$ TMA forms extended 2D networks of hydrogen bonded arrays yielding two types of nanoporous networks. The so-called "chicken-wire" and "flower" structures are based on hydrogen bonded dimers and trimers, respectively and are formed at the solution-solid ${ }^{11}$ as well as at the UHV-solid interface. ${ }^{10}$ The self-assembly of TMA has since served as a pioneering example of nanoporous networks ${ }^{12-14}$ which have been a subject of intense research. The nanoporous networks of TMA have been extensively utilized for immobilization of a variety of guest molecules ${ }^{10,14-18}$ and TMA has also served as a building block in multicomponent supramolecular architectures. ${ }^{19-24}$

TMA physisorbs with its phenyl ring parallel to the basal plane of graphite in order to maximize the interaction with the surface. In the chicken wire structure, all three carboxyl groups are hydrogen bonded with their neighbors via $\mathrm{R}_{2}^{2}(8)$ type hydrogen bonded dimers. The flower structure, on the other hand, is based on trimeric motifs that are hydrogen bonded via $\mathrm{R}^{3}{ }_{3}(12)$ type assembly. ${ }^{2}$ In both cases, the hydrogen bonded carboxyl groups are co-planar with the benzene ring upon adsorption on the graphite surface with the dihedral angle of $0^{\circ}$ (vide infra). The co-planarity of the carboxyl groups (and the resultant hydrogen bonds) with respect to the benzene ring ensures that molecule-substrate interactions are maximized. The planar adsorption with respect to the substrate coupled with the intermolecular hydrogen bonding interactions make TMA nanoporous networks extremely robust and reliable surface architectures. 
The presence of non-coplanarity however, does not necessarily prohibit self-assembled network formation. The steric aspects of hydrogen bonding for benzene carboxylic acids have been studied in the past but to a much lesser extent compared to the assembly of planar systems. For example, the lack of network formation for PA was explained by considering the steric hindrance between the two closely placed carboxyl groups that obstruct the formation of intermolecular hydrogen bonds. ${ }^{9}$ However, DFT calculations carried out on PA adsorbed on graphite later showed that the placement of the carboxyl groups and the resultant non-planarity do not necessarily prevent hydrogen bonding between the molecules and that the hydrogen bonding energy for PA dimers is comparable to that of ISA dimers. ${ }^{25}$ Benzene-1,2,4,5tetracarboxylic acid (pyromellitic acid, PMA), on the other hand, shows rich self-assembly behavior at the solution/graphite interface despite the non-coplanarity between the benzene ring and the closely spaced carboxyl groups. ${ }^{26}$

In this work, we re-visit the steric aspects of hydrogen bonding for benzene carboxylic acids by investigating the self-assembly of 2,4,6-triiodo-1,3,5-benzenetricarboxylic acid (ITMA, Figure 1) at the heptanoic acid/HOPG interface. Given the bulky nature of the iodine substituents on the benzene ring, we anticipated that the carboxyl groups will be rotated out-ofplane with respect to the benzene ring. Considering its structural resemblance with the intensively studied TMA, the molecular and the supramolecular structures of the two systems are compared using a joint theoretical-experimental approach. The self-assembled networks of I-TMA were characterized using STM and the energetics of the experimentally observed networks were gauged by carrying out detailed molecular modelling simulations. STM data reveal characteristic assembly behavior wherein I-TMA forms an auto-host-guest network. Furthermore, given their distinct hydrogen bonding ability, the on-surface mixing behavior of TMA with I-TMA was also studied. We demonstrate that, while the sterically enforced noncoplanarity does not obstruct the self-assembly of I-TMA itself, it prohibits mixing with TMA, which leads to the formation of supramolecular heterostructures. 


\section{Experimental methods}

\section{STM experiments}

I-TMA was synthesized via full iodination of mesitylene, followed by a permanganate oxidation of the three methyl groups as described in a patent. ${ }^{27}$ Slightly refined versions of this synthesis have been published recently. ${ }^{28,} 29$ TMA (Sigma-Aldrich, 98\%) and heptanoic acid (SigmaAldrich $\geq 99 \%$ ) were used without further purification. For STM experiments, stock solutions $(1 \mathrm{mg} / \mathrm{mL})$ of the solid compounds (TMA and I-TMA) were prepared in heptanoic acid. All STM experiments were performed at room temperature $\left(21-23^{\circ} \mathrm{C}\right)$ using a PicoLE (Keysight Technologies) machine operating in constant-current mode with the tip immersed in the supernatant liquid. STM tips were prepared by mechanically cutting a Pt/Ir wire $(80 \% / 20 \%$, diameter $0.2 \mathrm{~mm}$ ). Prior to imaging, a drop of the solution was placed onto a freshly cleaved surface of highly oriented pyrolytic graphite (HOPG, grade ZYB, Advanced Ceramics Inc., Cleveland, USA). The experiments were repeated in 2-3 sessions using different tips to check for reproducibility and to avoid experimental artefacts, if any. For analysis purposes, recording of a monolayer image was followed by imaging the graphite substrate under the same experimental conditions, except for increasing the current and lowering the bias. The images were corrected for drift via Scanning Probe Image Processor (SPIP) software (Image Metrology ApS), using the recorded graphite images for calibration purposes, allowing a more accurate unit cell determination. The unit cell parameters were determined by examining at least 4 images and only the average values are reported. The images are Gaussian filtered. The imaging parameters are indicated in the figure caption: tunneling current $\left(\mathrm{I}_{\text {set }}\right)$, and sample bias $\left(\mathrm{V}_{\text {bias }}\right)$.

\section{COMPUTATIONAL METHODS}

\section{STM simulations}

For the simulation of the STM simulated, all DFT calculations were performed using the VASP $\operatorname{code}^{30}$ with the projector-augmented wave (PAW) approach and a kinetic energy cutoff of 400 
$\mathrm{eV}$ using a Gamma point sampling of $2 \times 2 \times 1$ for the Brillouin zone (BZ) integration. The exchange and correlation effects were treated with the Perdew-Burke-Ernzerhof (PBE) functional ${ }^{31}$ incorporating the dispersion forces by Grimme correction $(\mathrm{PBE}+\mathrm{D} 2)^{32}$ to account for the van der Waals (vdW) interactions. All DFT/SCF calculations were performed on the geometries/structures as obtained from molecular dynamics simulations and no further optimization was performed. The simulated STM images were calculated considering the conduction band electronic structure including the contributions from oxygen and iodine atoms, such that the chosen energy window range is close the experimental protocols.

\section{MM and MD simulations}

Force field-based simulations were conducted with the BIOVIA Materials Studio 2018 molecular modeling package, ${ }^{33}$ using its implementation of the PCFF force field, which extends the original CFF91 force field. ${ }^{34}$ For the NVT simulations, the temperature was controlled with the Nose-Hoover thermostat, ${ }^{35}$ while in the NPT simulations, the pressure was controlled with the Parrinello barostat. ${ }^{36}$ Atomic charges from the force field were used for the solvent molecules, as well as for I-TMA and TMA. The graphite surface was modelled using two graphite layers considered as rigid bodies, since molecular physisorption does not affect the surface structure in any meaningful way. While the graphite surface was present during the MD simulations, its energetic contribution to the system was removed from the analysis. This way, we could calculate the interactions of the aggregates with the solvent molecules, $E_{\text {int(monolayer- }}$ solvent), without considering the "energetic noise" coming from the solvent-surface interactions.

\section{Results and discussion:}

\section{Conformation of a single I-TMA molecule}

In order to understand the orientation of the carboxyl groups with respect to the plane of the phenyl ring, quantum chemistry calculations were employed to compute the geometry of isolated 
I-TMA molecules. Similar calculations were also carried out for TMA for the sake of comparison. For both the systems, the geometry was optimized with the Gaussian $09 \operatorname{code}^{37}$ at DFT B3LYP 6-31g** theory level (the LANL2DZ basis $\operatorname{set}^{38}$ was used to describe the iodine atoms of I-TMA), while the atomic charges were calculated at CM5 level. ${ }^{39}$ Figure 1 shows the differences in geometries of single molecules of I-TMA and TMA. As anticipated, TMA exhibits a fully flat conformation where the carboxyl groups remain coplanar with the molecular core. The carboxyl groups of I-TMA on the other hand, are tilted $90^{\circ}$ with respect to the benzene ring due to the steric hindrance of the iodine atoms. Frequency calculations (see supporting information section 2) show that the structures reported here are the true minima for the molecular geometries of isolated I-TMA and TMA.
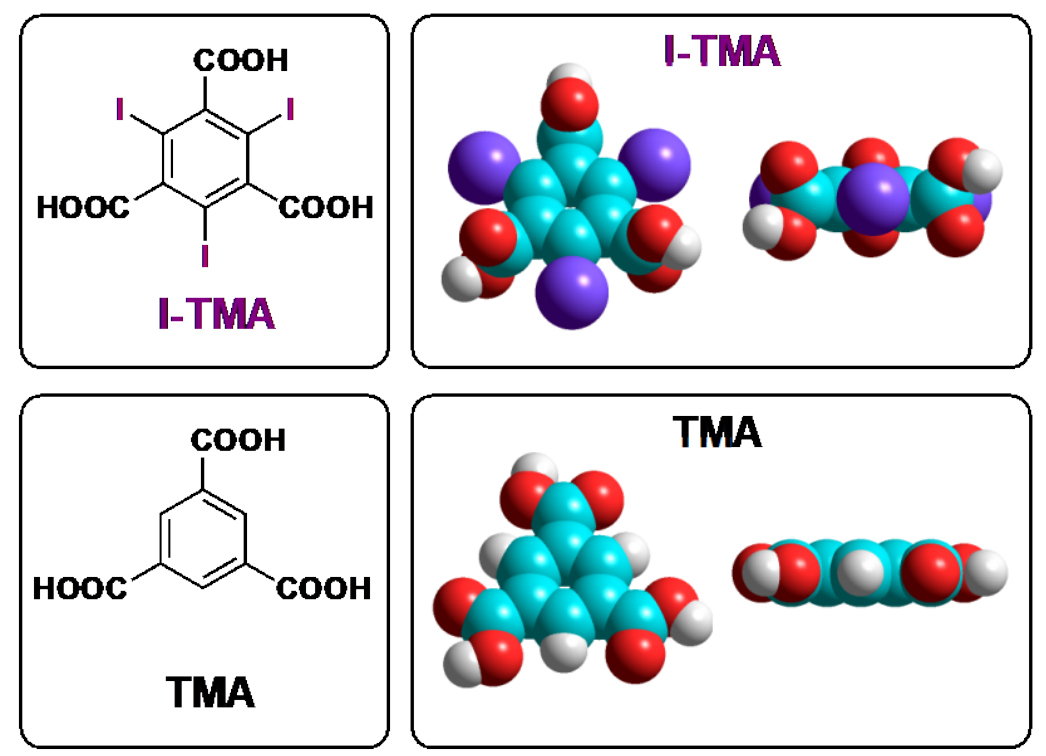

Figure 1: Molecular structures and geometry of iodinated trimesic acid (I-TMA) and trimesic acid (TMA).

To further understand the behavior of these molecules upon adsorption on the basal plane of graphite, a force field-based molecular modeling approach was used wherein molecular mechanics (MM) and molecular dynamics (MD) simulations were carried out. The PCFF force field ${ }^{34}$ as implemented in BIOVIA's molecular modeling package Materials Studio $2018^{33}$ was 
chosen as it has been parameterized for halogens and is able to fully reproduce the geometry of isolated molecules obtained from the DFT calculations. These simulations revealed that when adsorbed on the surface of graphite, the I-TMA molecule does not undergo planarization. The carboxyl groups remain tilted at $90^{\circ}$ with respect to the central benzene ring. In the case of TMA, the carboxyl groups remain co-planar with respect to the benzene ring. The adsorption distance, i.e., the distance between the center of the benzene ring and the graphite surface, is $3.9 \AA$ for ITMA versus $3.4 \AA$ for TMA as expected from the non-coplanar geometry of the former. Contrary to the expectation however, the adsorption energy of I-TMA (-29.8 kcal mol-1) was found to be comparable to that for TMA $\left(-29.5 \mathrm{kcal} \mathrm{mol}^{-1}\right)$ despite the more planar adsorption of the latter. This could be due to the higher polarizability, and the larger footprint of I-TMA which leads to higher van der Waals contact with the surface compared to that for TMA.

\section{Self-assembly at the solution/graphite interface}

The self-assembly behavior of I-TMA at the solution/solid interface was studied using STM. Figure 2a shows the large scale STM image of the I-TMA self-assembled monolayer physisorbed at the heptanoic acid/HOPG interface. The surface is covered with multiple domains made up of triangular features. It can be readily noticed that the triangular features are differently oriented throughout the surface and the orientation appears to be domain specific. Figure $2 \mathrm{a}$ shows a prominent domain border that runs approximately through the middle of the STM image and appears as a dark trench-like feature. Apart from these obvious domain borders, the surface shows peculiar thread-like structures (yellow arrows) that separate the upward and the downward facing triangular features from each other. In comparison to TMA, the self-assembled monolayer of I-TMA was found to be rather fragile to STM imaging. Furthermore, the structure of the monolayer of I-TMA does not match with the typical honeycomb and flower structures reported for TMA. ${ }^{10,11}$ 
In order to understand the basis of molecular self-assembly of I-TMA in terms of intermolecular interactions, high-resolution STM images of the monolayer were obtained. Figure $\mathbf{2 b}$ shows a Gaussian filtered high-resolution STM image of the I-TMA monolayer. Each triangular feature (marked by a white hexagon) consists of a cluster of nine bright dots whereas the center of the triangle appears dark. The clusters constitute the repeat units of the network and are separated from each other by dark areas. We attribute the bright dots to the iodine atoms of I-TMA molecules. It follows that the STM contrast is dominated by iodine atoms. STM simulations presented in Figure 3e confirm the assignment of the STM contrast. This is also in line with literature where iodine atoms have been reported to appear brighter compared to the aromatic as well as the aliphatic backbones of molecules. ${ }^{40-42}$ The unit cell parameters obtained from calibrated STM images match closely with those of TMA honeycomb network (Table 1). Note that with similar unit cell parameters, TMA forms an open porous network whereas the I-TMA network consists of densely packed molecules. This observation is attributed to an auto-hostguest network, where I-TMA forms a hydrogen bonded hexameric network similar to TMA with cavities occupied by I-TMA molecules which also serve as guests. Figure $2 \mathrm{c}$ shows a tentative model for such auto-host-guest system which assumes a perfect honeycomb network for I-TMA, the cavities of which contain immobilized I-TMA molecules. Note that, while the model seems to agree reasonably well with the STM image, differences exist. Specifically, the distances between the iodine atoms do not match perfectly with those in the STM images. Furthermore, a close inspection of the STM image also reveals that the host structure, a large part of which is not visible in STM images, may actually consist of a distorted hexagonal network instead of the regular honeycomb depicted in the model presented in Figure 2c. 

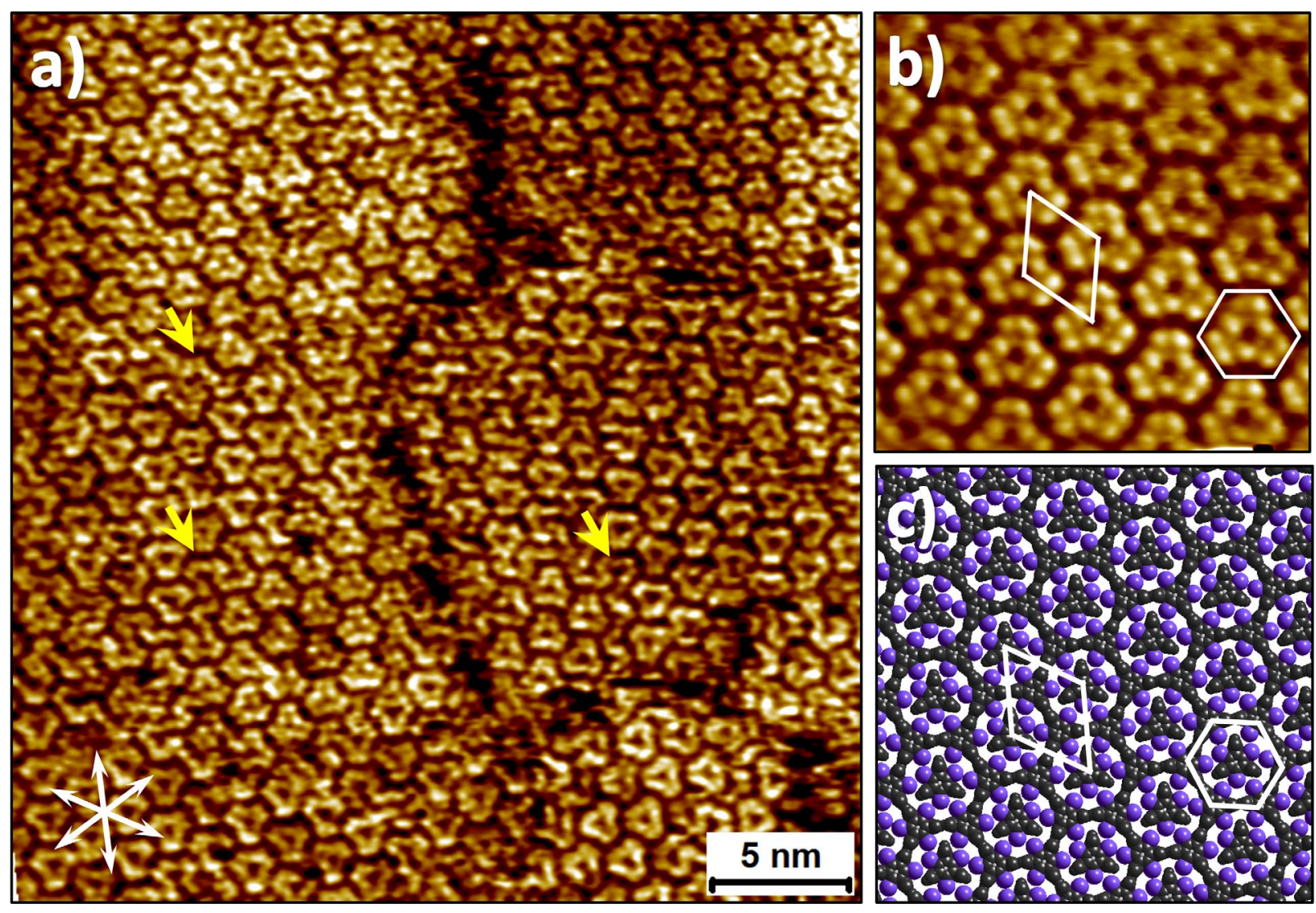

Figure 2. Self-assembly of I-TMA at the heptanoic acid/HOPG interface. (a) Large-scale STM image of the network. The yellow arrows indicate thread-like features that correspond to the domain boundaries and arise due to random orientation of the molecules. (b) High-resolution STM image $(8.3 \mathrm{~nm} \times 8.3 \mathrm{~nm})$. (c) Proposed molecular model showing the arrangement of ITMA molecules within the self-assembled network. Since the contrast in the STM images is dominated by iodine atoms, the molecular model is presented with $\mathrm{C}, \mathrm{H}$ and $\mathrm{O}$ in black and iodine atoms in purple so that the features observed in the STM images match closely with those in the proposed molecular model. See Figure 3 for molecular models presented in standard colors. Imaging parameters: $I_{\text {set }}=110 \mathrm{pA}, V_{\text {bias }}=750 \mathrm{mV}$. For additional STM images see Figure $\mathrm{S} 1$ in the supporting information. 
Table 1. Structural (STM, MM) and Energetic (force field calculations) Parameters of the TMA (honeycomb) and I-TMA Networks.

\begin{tabular}{|c|c|c|c|c|c|c|c|c|c|c|}
\hline & \multicolumn{3}{|c|}{$\begin{array}{c}\text { Unit cell parameters } \\
\text { (experimental) }\end{array}$} & \multicolumn{3}{|c|}{$\begin{array}{l}\text { Unit cell parameters } \\
\text { (calculated) }\end{array}$} & \multirow{2}{*}{$\begin{array}{c}\begin{array}{c}\mathrm{E}_{\mathrm{ads}} \\
(\mathrm{kcal} / \mathrm{mol})\end{array} \\
\begin{array}{c}\text { Single } \\
\text { molecule }\end{array}\end{array}$} & \multirow{2}{*}{$\begin{array}{c}E_{\mathrm{ads}} \\
(\mathrm{kcal} / \mathrm{mol}) \\
\text { Hexamer }\end{array}$} & \multirow{2}{*}{$\begin{array}{c}\mathrm{E}_{\text {int }} \\
(\mathrm{kcal} / \mathrm{mol}) \\
\begin{array}{c}\text { Molecule- } \\
\text { solvent }\end{array} \\
\end{array}$} & \multirow{2}{*}{$\begin{array}{c}\mathrm{E}_{\mathrm{int}} \\
(\mathrm{kcal} / \mathrm{mol}) \\
\text { Monolayer- } \\
\text { solvent }\end{array}$} \\
\hline & $\mathrm{a}(\mathrm{nm})$ & $\mathrm{b}(\mathrm{nm})$ & $\gamma\left({ }^{\circ}\right)$ & $\mathrm{a}(\mathrm{nm})$ & $\mathrm{b}(\mathrm{nm})$ & $\gamma\left({ }^{\circ}\right)$ & & & & \\
\hline TMA & $1.6 \pm 0.1$ & $1.6 \pm 0.1$ & $59 \pm 1$ & 1.7 & 1.7 & 59 & -29.5 & -189 & -78.3 & -206.7 \\
\hline I-TMA & $1.6 \pm 0.1$ & $1.6 \pm 0.1$ & $61 \pm 2$ & 1.7 & 1.7 & 60 & -29.8 & -204 & -87.4 & -231.7 \\
\hline
\end{tabular}

\section{MM and MD simulations of the self-assembled networks}

To understand the structure of the network formed by I-TMA in detail, the self-assembly was modelled using a combination of MD and MM simulations. First a hexameric hydrogen bonded unit of I-TMA was built and its geometry was optimized on the surface of graphite (Figure 3a). The honeycomb unit of TMA was also built for the sake of comparison (Figure 3b). The simulations revealed that the unit cell of the I-TMA honeycomb is in close agreement with that observed experimentally. Furthermore, it also matches closely with that of the TMA honeycomb network (Table 1). Despite the identical unit cell, the hexamer of I-TMA differs from that of TMA in two aspects. Firstly, the hydrogen bonded carboxyl groups are coplanar with respect to the benzene ring in the case of TMA whereas they are orthogonal for I-TMA hexamer. Such edge-on orientation of the carboxyl groups promotes the formation of an intermolecular H-bonding network that allows more flexibility in the relative position of I-TMA molecules. This is in agreement with the observation of the I-TMA hexamer being a slightly distorted hexagon compared to that formed by TMA, which exhibits a perfect hexagonal shape. Secondly, due to its larger footprint and also due to the protruding iodine atoms, the cavity size of I-TMA is smaller than that of TMA. The pore diameter $(d)$ obtained from the simulated structures is $15.6 \AA$ for TMA compared to only $12.2 \AA$ for I-TMA (Figure 3a, b). Furthermore, once again contrary to our expectation, the I-TMA hexamer $\left(-204 \mathrm{kcal} \mathrm{mol}^{-1}\right)$ was found to be more stable than the TMA hexamer $\left(-189 \mathrm{kcal} \mathrm{mol}^{-1}\right)$. 

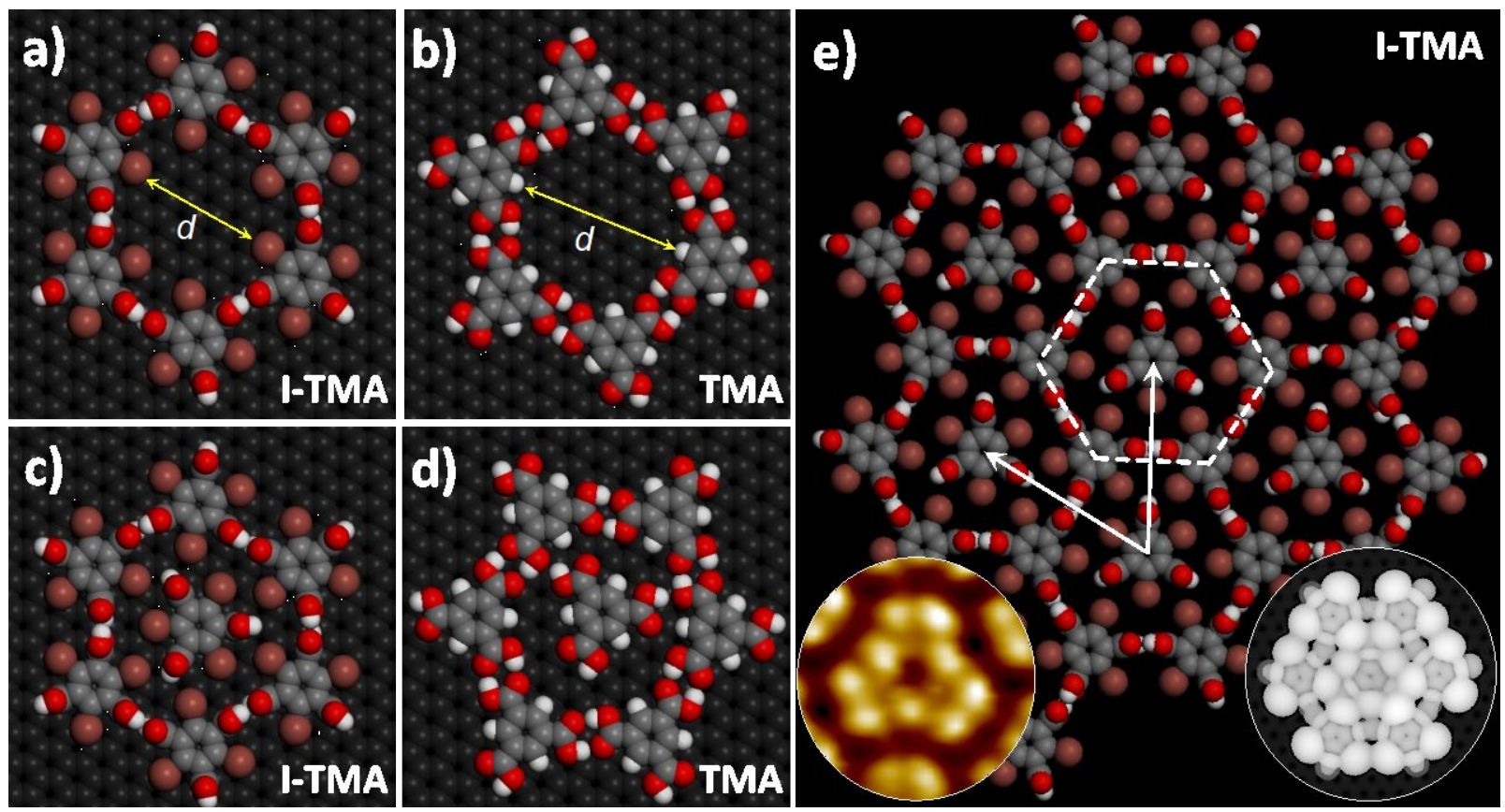

Figure 3. Simulated molecular models for the hexameric host networks $(a, b)$ and auto-hostguest networks (c, d) for I-TMA and TMA. (e) An extended optimized model for I-TMA selfassembly. The inset in the lower left corner shows a simulated STM image of a portion of the model highlighted by dotted white line (7 I-TMA molecules). A digital zoom of a highresolution STM image is provided in the lower left corner for the sake of comparison.

It is noteworthy that despite forming identical hexameric host cavities, I-TMA exclusively forms an auto-host-guest network whereas TMA, under a wide variety of experimental conditions prefers to form either a honeycomb or a flower network, with empty cavities. Auto-host-guest networks for TMA are reported rarely. ${ }^{43}$ The much higher propensity to trap the guest molecule in the case of I-TMA arises from the smaller and somewhat oddly shaped pores formed within the network. These pores offer a perfect cavity for the immobilization of another I-TMA molecule based on size and shape complementarity (Figure $3 \mathrm{c})$. The formation of a halogen bond between the iodine atoms of the guest and the oxygen atoms of the host network (and vice versa) can be ruled out based on the distances measured from molecular models which are larger than the sum of van der Waals radii of the concerned 
atoms. The TMA pore on the other hand, is larger than the ideal cavity needed to immobilize another molecule of TMA inside (Figure 3d). This is also reflected in the calculated strengths of interactions of the TMA and I-TMA guest molecules with their respective hexameric aggregates. The I-TMA guest molecule interacts with its host network more than twice as strongly as that in the case of TMA $\left(-107.3 \mathrm{kcal} \mathrm{mol}^{-1}\right.$ for I-TMA versus $-48.0 \mathrm{kcal} \mathrm{mol}^{-1}$ for TMA). The tighter fit and the consequent stronger interaction with the pore clearly explains the exclusive formation of auto-host-guest network in the case of I-TMA. Finally, STM simulations were carried out on an auto-host-guest cluster presented in Figure 3c to confirm the initial assumption that the STM contrast is dominated by iodine atoms. The inset in Figure 3e shows the simulated STM image which closely reproduces the cluster of bright features observed in high-resolution STM images. Given that the STM contrast mostly arises from the iodine atoms, the thread-like features noticeable in large scale STM images (see for example, Figure 2a) can now be understood as rotational defects related to the random orientation of the I-TMA molecules.

\section{Mixing behavior of TMA and I-TMA: Van der Waals supramolecular heterostructures}

Having understood the structural and energetic aspects of I-TMA self-assembly, we now describe the mixing behavior of I-TMA and TMA. Since the two molecules crystallize into identical unit cells, it was anticipated that one type would insert into the self-assembled network of the other in a random fashion without disturbing its unit cell. ${ }^{44}$ It remained to be seen however, if the non-coplanarity of the phenyl ring and the carboxyl groups in the case of I-TMA would prohibit such intimate mixing as anticipated. The mixing experiments were carried out both using premixed solutions with defined stoichiometry and also via sequential addition of the two components on the graphite surface. The results described below are the same irrespective of the method of deposition. Furthermore, for the sequential deposition protocol, the results described below remain the same irrespective of the order of deposition of the two components. 
Figure 4 shows the surface of HOPG after sequential addition of heptanoic acid solutions of TMA and I-TMA. The STM image in Figure 4a clearly shows an incomplete monolayer of ITMA formed on top of a seemingly complete monolayer of TMA. This means that the two molecules neither mix nor undergo phase separation in $2 \mathrm{D}$ but rather phase separate orthogonal to the substrate leading to the formation of a supramolecular heterostructure. Comparison of the I-TMA monolayer formed on top of the TMA monolayer with that formed directly on the HOPG substrate (see Figure 2a) reveals that the former is virtually free of the rotational defects described earlier (yellow arrows, Figure 2a). A number of high-resolution STM images indicate that the TMA monolayer is indeed underneath the I-TMA monolayer. Very often, the TMA network can be seen underneath the exposed areas/defects in the I-TMA monolayer (Figure 4b, d), or isolated islands of I-TMA can be observed formed on the top of a continuous TMA monolayer (Figure 4c). The better degree of order in the I-TMA network when formed atop TMA network indicates relatively better dynamics during the assembly process. Furthermore, the unit cells of the two networks are perfectly aligned and can be superimposed indicating epitaxial alignment between the molecules adsorbed in the respective layers (see Figure S3 in the supporting information). The near perfect registry between the two networks arises in part from the matching periodicities of the I-TMA and the TMA structures.

The lack of mixing of the two components can be traced back to the non-coplanarity of the carboxyl groups of I-TMA and in turn, to the difference in the H-bonding behavior of the two molecules. It can be readily understood that the carboxyl groups of TMA need to rotate outof-plane in order to hydrogen bond with those of I-TMA. Such out-of-plane twisting of carboxyl groups will be associated with the consequent decrease in the molecule-substrate interactions for TMA. Such heteromolecular TMA/I-TMA dimer is expected to be energetically less stable compared to the homomolecular TMA dimer. Note that the co-crystallization of two components is favored only when the resulting structure is lower in energy and thus more stable compared to the native structures of individual components. Therefore, in the present case, the two molecules 

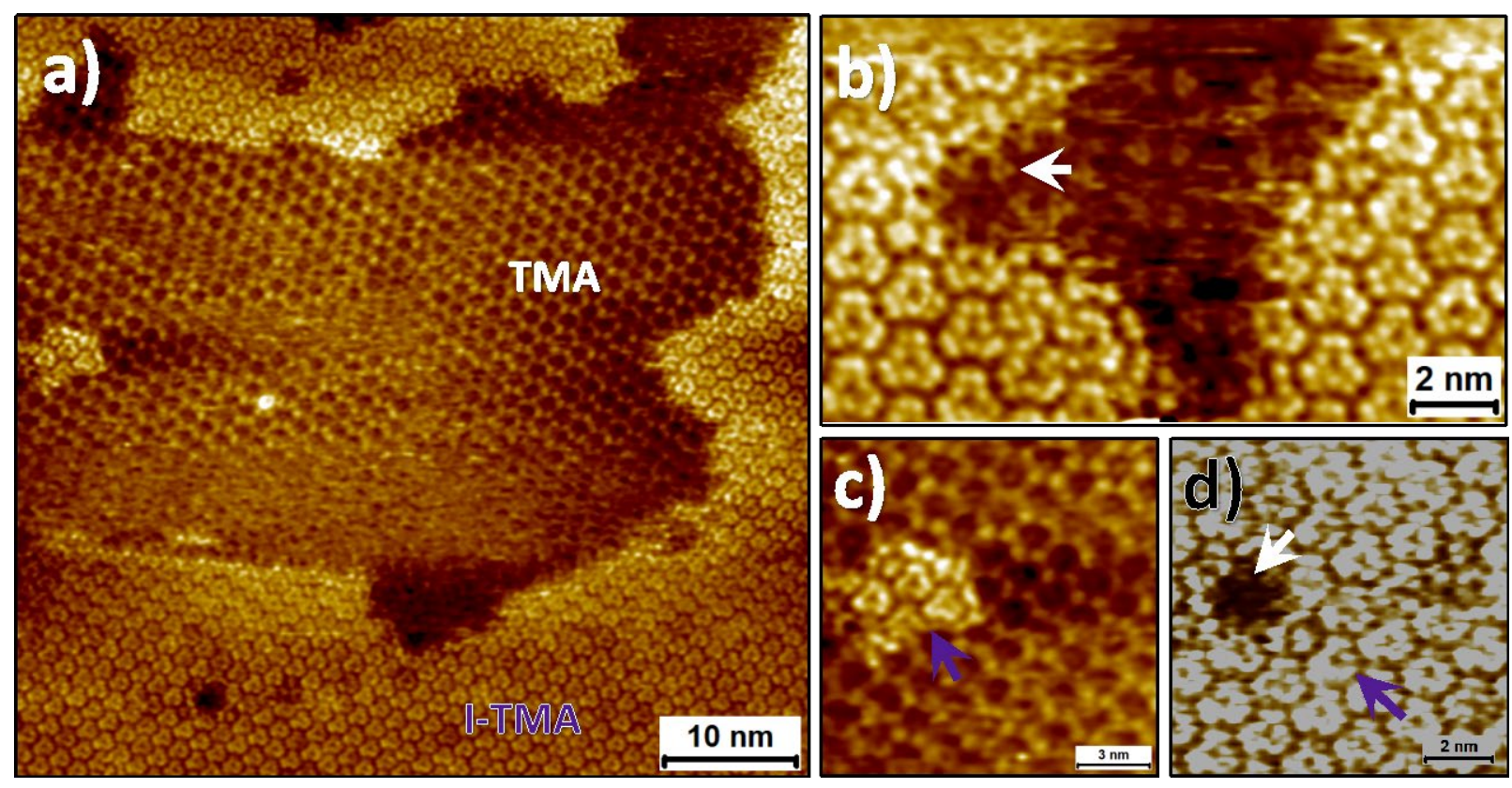

Figure 4. Heteromolecular bilayers formed at the heptanoic acid/HOPG interface by a mixture of TMA and I-TMA. (a) STM image showing an incomplete monolayer of I-TMA formed atop TMA monolayer. Note that the I-TMA monolayer, though incomplete, is virtually defect free unlike the I-TMA monolayer formed directly on the surface of HOPG. (b-d) STM images providing an evidence of the bilayer formation. TMA monolayer (white arrow) can be seen underneath and adjacent to the incompletely formed I-TMA monolayer in (b). Panel (c) shows an island of I-TMA (purple arrow) adsorbed on top of the honeycomb network of TMA. (d) shows the self-assembled TMA hexagon (white arrow) through a defect formed in the top ITMA monolayer (purple arrow). Imaging parameters: (a, b): $I_{\text {set }}=110 \mathrm{pA}, V_{\text {bias }}=-700 \mathrm{mV}$, (c, d): $I_{\text {set }}=70 \mathrm{pA}, V_{\text {bias }}=-1200 \mathrm{mV}$. For additional STM images see Figure S2 in the supporting information.

prefer to undergo phase separation instead of random mixing or stoichiometric co-crystallization on the surface. It is important to note that despite the possibility of the immobilization of a single I-TMA molecule within the cavities of the TMA network, no host-guest complexes were ever observed. While the preferential formation of a supramolecular bilayer instead of in-plane phase separation can be understood based on the aforementioned arguments, the preference for the 
TMA monolayer to adsorb as the bottom layer is not obvious, especially given the comparable adsorption enthalpies of the two molecules (Table 1).

\section{MM and MD simulations of the supramolecular heterostructures}

In order to gain insight into the TMA/I-TMA supramolecular heterostructure, especially to understand the preference of I-TMA to adsorb atop the TMA monolayer, which implies a close contact with the solvent, the interaction energies of the two molecules with the solvent, namely heptanoic acid, were estimated using MD simulations. In order to validate the ability of the PCFF force field to reproduce the intermolecular interactions between the molecules of heptanoic acid, a periodic box containing 128 molecules of heptanoic acid was built. MD simulations were then conducted in the NPT ensemble (constant number of particles, pressure and temperature) to calculate the liquid density. The theoretical density at the end of a 5-ns long $\mathrm{MD}$ run at $300 \mathrm{~K}$ and $1 \mathrm{~atm}$ was found to be $0.91 \mathrm{~g} / \mathrm{cm}^{3}$. This value is in perfect agreement with the experimental density of $0.918 \mathrm{~g} / \mathrm{cm}^{3}$ thus justifying the use of PCFF force field.

After confirming the adequacy of PCFF force field for describing the solvent, the affinity of single TMA and I-TMA molecules with heptanoic acid was calculated. This was done by soaking the respective single molecules into a heptanoic acid solvent box (Figure S4 in the supporting information). The solute-solvent interaction energy, $E_{\text {int }}$ (molecule-solvent) was then calculated as the energy difference between the total energy of the system, $E_{\text {sys }}$ and the sum of the energy of the isolated molecule, $\mathrm{E}_{\mathrm{mol}}$, and solvent box, $\mathrm{E}_{\mathrm{sol}}$ :

$$
E_{\text {int(molecule-solvent })}=E_{\text {sys }}-\left(E_{\text {mol }}+E_{\text {sol }}\right)
$$

The interaction energies were calculated at the end of several $1 \mathrm{~ns}-$ long MD simulations in the NVT ensemble (constant number of particles, volume and temperature) at $300 \mathrm{~K}$, to allow the system to equilibrate thermally. As anticipated, these simulations revealed that hydrogen bonds are formed between the carboxyl groups of heptanoic acid and those of TMA as well as I-TMA. 
No hydrogen bond-like interaction was observed between the solvent molecules and the iodine atoms of I-TMA was observed. Most importantly, these calculations show that heptanoic acid has a more favorable interaction with I-TMA $\left(E_{\text {int(molecule-solvent })}=-87.4 \mathrm{kcal} \mathrm{mol}^{-1}\right)$ than TMA $\left(E_{\text {int }(\text { molecule-solvent })}=-78.3 \mathrm{kcal} \mathrm{mol}^{-1}\right)$. This $9 \mathrm{kcal} \mathrm{mol-1}$ difference in the solute-solvent interaction energies is consistent with the observation that I-TMA prefers to adsorb on top of the TMA monolayer, at the interface with the solvent. Furthermore, it is plausible that this $9 \mathrm{kcal}$ $\mathrm{mol}^{-1}$ difference in the interaction with the solvent supersedes the identical adsorption energies of I-TMA and TMA on graphite.

It can be argued however that the two monolayers are structurally different and thus the interaction energies of single molecules with the solvent may not provide sufficient description of the observed behavior. This is because the TMA monolayer consists of a porous selfassembled network and thus incorporates solvent molecules in its hexagonal cavities. The ITMA network, in contrast, is non-porous. To get comprehensive insight into this aspect, the actual monolayer-solvent interface was modelled in order to test whether the better molecule/solvent interaction observed for the single molecules of I-TMA relative to TMA is maintained when the molecules are part of a monolayer. To keep the computational cost manageable, the interface between the solvent layer and a single I-TMA (7 molecules) or TMA (6 molecules) aggregate adsorbed on HOPG was modelled (Figure S5 in the supporting information).

The monolayer-solvent interaction energy $E_{\text {int(monolayer-solvent) }}$ was calculated as the difference between the total energy of the solvated aggregate, $\mathrm{E}_{\mathrm{sys}}$, and the sum of the energy of the non-interacting solvent phase, $\mathrm{E}_{\text {solv }}$, and molecular aggregate, $\mathrm{E}_{\text {agg: }}$ :

$$
E_{\text {int }(\text { monolayer-solvent })}=E_{\text {sys }}-\left(E_{\text {solv }}+E_{\text {agg }}\right)
$$

These calculations further corroborated the results obtained for single molecules. The ITMA monolayer was found to have a more favorable interaction $\left(E_{\text {int }(\text { monolayer-solvent }}\right)=-231.7 \mathrm{kcal}$ 
mol $\left.^{-1}\right)$ with the solvent than the TMA monolayer $\left(E_{\text {int(monolayer-solvent })}=-206.7 \mathrm{kcal} \mathrm{mol}^{-1}\right)$. The difference in the interaction energies with the solvent for the two systems $\left(25 \mathrm{kcal} \mathrm{mol}^{-1}\right)$ is again consistent with the observed preference of the I-TMA monolayer to be located at the interface with the solvent. The results described above are obviously specific for heptanoic acid and it remains to be seen if the mixing behavior of the two molecules will be any different in other solvents.

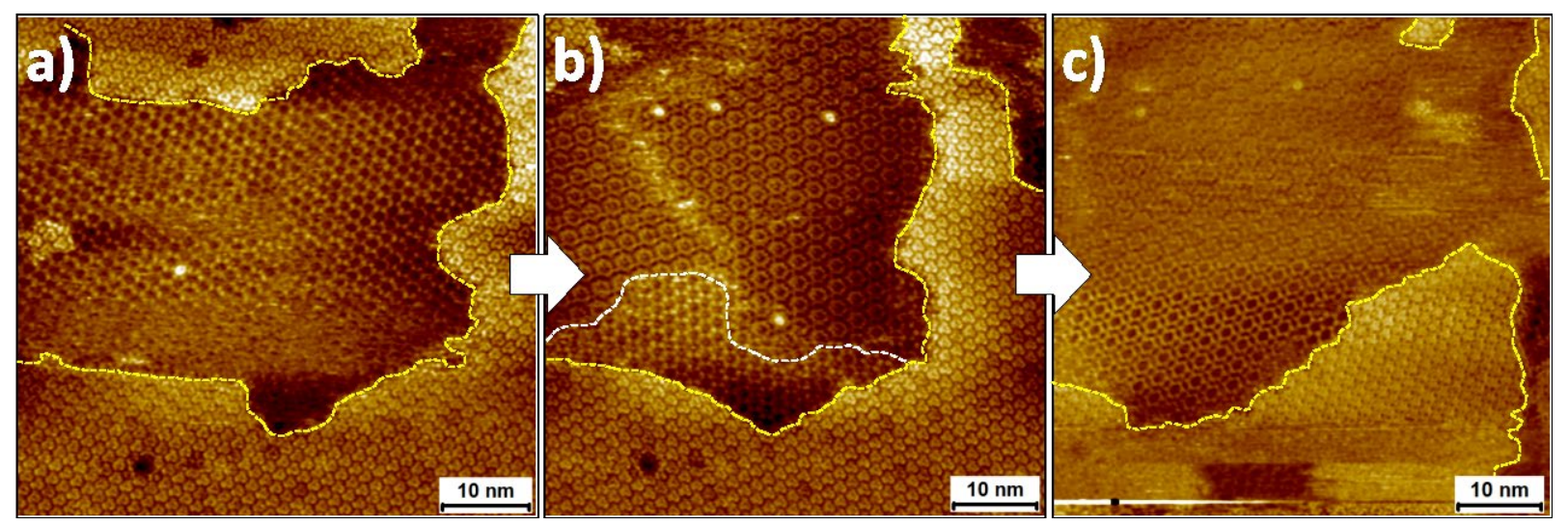

Figure 5. Desorption dynamics in TMA/I-TMA supramolecular heterostructure. (a-c) Sequential STM images showing the gradual desorption of the I-TMA layer residing on top of a bottom TMA honeycomb network as the latter transitions into the flower structure. The scale bar $=10 \mathrm{~nm}$.

\section{Phase transition/desorption dynamics}

An interesting phase transition dynamic was observed during the STM image acquisition of the TMA/I-TMA supramolecular heterostructure. Figure 5 shows three sequential STM images obtained by scanning approximately the same area. The bottom TMA layer transitions from the honeycomb into the flower structure as a function of time. This transition is associated with the concomitant desorption of the I-TMA layer from the top as evident from the STM images provided in Figure 5a-c. These observations clearly suggest that the TMA/I-TMA supramolecular heterostructure is stable only when the templating TMA layer exists as a 
honeycomb network. Note that the flower structure of TMA has a larger unit cell compared to that of both honeycomb TMA and the close packed I-TMA network (see Table 1) and thus may not offer the same extent of stabilization as the honeycomb network of TMA. This could explain, at least in part, the removal of the I-TMA network as the bottom layer transitions into a flower structure.

\section{Conclusions:}

Hydrogen bonds, especially those formed between two carboxylic groups, remain one of the archetypal examples of robust supramolecular interactions. In the work described above, we have reported on the unusual self-assembly behavior of an aromatic tricarboxylic acid in which the carboxyl groups and thus the resultant hydrogen bonds are forced to be orthogonal to the central benzene ring. The STM data revealed that I-TMA exclusively forms an auto-host-guest network at the solution-solid interface. Detailed molecular modeling simulations show that the tendency to form the experimentally observed auto-host-guest network arises from the perfect size and shape complementarity between single I-TMA molecules and the host cavities of the I-TMA network. This results in stronger binding of the guest molecules to the host cavities in the case of I-TMA relative to the case of TMA. The non-coplanarity of the carboxyl groups of I-TMA led to peculiar mixing behavior with TMA at the solution-solid interface. A supramolecular heterostructure, in which the planar TMA forms a monolayer in contact with the substrate, and I-TMA monolayer on top of the first layer was formed. The simulations suggest that I-TMA forms the top layer of the heterostructure despite having comparable adsorption energy on graphite with respect to TMA, due to its preferential solvation in heptanoic acid which outweighs the adsorption energy. It remains to be seen if the mixing behavior is any different in other solvents.

The work presented above shows that despite being intensively studied and reported for more than a decade, the on-surface self-assembly of aromatic carboxylic acids is a rich domain 
for studying the fundamentals of molecular self-assembly. It remains to be seen how the noncoplanarity of the carboxyl groups and the presence of the bulky iodine atoms on the backbone affect the crystallization behavior in bulk (solid state) and the self-assembly in the solution phase. The supramolecular heterostructure formed upon mixing TMA and I-TMA represents a unique example of phase separation orthogonal to the surface where the two layers are stabilized by inplane and out-of-plane hydrogen bonds, respectively.

\section{SUPPORTING INFORMATION}

Supporting Information Available: The Supporting Information is available free of charge on the ACS Publications website at DOI:

Additional STM images and the computational details (File format: PDF).

\section{ACKNOWLEDGMENTS}

The authors gratefully acknowledge financial support from the Fund of Scientific Research Flanders (FWO), KU Leuven - Internal Funds. This work was in part supported by FWO and FNRS under grant EOS 30489208 (2Dto3D project). The molecular modeling activities are supported by FNRS (Consortium des Équipements de Calcul Intensif - CÉCI, under Grant 2.5020.11) and by the Walloon Region (ZENOBE Tier-1 supercomputer, under grant 1117545).

\section{References:}

1. Steiner, T. The Hydrogen Bond in the Solid State. Angew. Chem. Int. Ed. 2002, 41, 4876.

2. Ivasenko, O.; Perepichka, D. F. Mastering Fundamentals of Supramolecular Design with Carboxylic Acids. Common Lessons from X-Ray Crystallography and Scanning Tunneling Microscopy. Chem. Soc. Rev. 2011, 40, 191-206. 
3. Goronzy, D. P.; Ebrahimi, M.; Rosei, F.; Arramel; Fang, Y.; De Feyter, S.; Tait, S. L.; Wang, C.; Beton, P. H.; Wee, A. T. S., et al. Supramolecular Assemblies on Surfaces: Nanopatterning, Functionality, and Reactivity. ACS Nano 2018, 12, 74457481.

4. Slater, A. G.; Perdigão, L. M. A.; Beton, P. H.; Champness, N. R. Surface-Based Supramolecular Chemistry Using Hydrogen Bonds. Acc. Chem. Res. 2014, 47, 34173427.

5. Lackinger, M.; Heckl, W. M. Carboxylic Acids: Versatile Building Blocks and Mediators for Two-Dimensional Supramolecular Self-Assembly. Langmuir 2009, 25, 1130711321.

6. Korolkov, V. V.; Baldoni, M.; Watanabe, K.; Taniguchi, T.; Besley, E.; Beton, P. H. Supramolecular Heterostructures Formed by Sequential Epitaxial Deposition of TwoDimensional Hydrogen-Bonded Arrays. Nat. Chem. 2017, 9, 1191-1197.

7. Korolkov, V. V.; Allen, S.; Roberts, C. J.; Tendler, S. J. B. Green Chemistry Approach to Surface Decoration: Trimesic Acid Self-Assembly on Hopg. J. Phys. Chem. C 2012, $116,11519-11525$.

8. Cyr, D. M.; Venkataraman, B.; Flynn, G. W. Stm Investigations of Organic Molecules Physisorbed at the Liquid-Solid Interface. Chem. Mater. 1996, 8, 1600-1615.

9. Lackinger, M.; Griessl, S.; Markert, T.; Jamitzky, F.; Heckl, W. M. Self-Assembly of Benzene-Dicarboxylic Acid Isomers at the Liquid Solid Interface: Steric Aspects of Hydrogen Bonding. J. Phys. Chem. B 2004, 108, 13652-13655.

10. S. Griessl, M. L., M. Edelwirth, M. Hietschold and W. M. Heckl Self-Assembled TwoDimensional Molecular Host-Guest Architectures from Trimesic Acid. Single Mol. 2002, $3,25-31$. 
11. Lackinger, M.; Griessl, S.; Heckl, W. M.; Hietschold, M.; Flynn, G. W. Self-Assembly of Trimesic Acid at the Liquid-Solid Interface a Study of Solvent-Induced Polymorphism. Langmuir 2005, 21, 4984-4988.

12. Kudernac, T.; Lei, S.; Elemans, J. A. A. W.; De Feyter, S. Two-Dimensional Supramolecular Self-Assembly: Nanoporous Networks on Surfaces. Chem. Soc. Rev. 2009, 38, 402-421.

13. Elemans, J. A. A. W.; Lei, S.; De Feyter, S. Molecular and Supramolecular Networks on Surfaces: From Two-Dimensional Crystal Engineering to Reactivity. Angew. Chem. Int. Ed. 2009, 48, 7298-7332.

14. Teyssandier, J.; Feyter, S. D.; Mali, K. S. Host-Guest Chemistry in Two-Dimensional Supramolecular Networks. Chem. Commun. 2016, 52, 11465-11487.

15. Griessl, S. J. H.; Lackinger, M.; Jamitzky, F.; Markert, T.; Hietschold, M.; Heckl, W. M. Incorporation and Manipulation of Coronene in an Organic Template Structure. Langmuir 2004, 20, 9403-9407.

16. Griessl, S. J. H.; Lackinger, M.; Jamitzky, F.; Markert, T.; Hietschold, M.; Heckl, W. M. Room-Temperature Scanning Tunneling Microscopy Manipulation of Single C60 Molecules at the Liquid-Solid Interface: Playing Nanosoccer. J. Phys. Chem. B 2004, $108,11556-11560$.

17. Ivasenko, O.; MacLeod, J. M.; Chernichenko, K. Y.; Balenkova, E. S.; Shpanchenko, R. V.; Nenajdenko, V. G.; Rosei, F.; Perepichka, D. F. Supramolecular Assembly of Heterocirculenes in 2d and 3d. Chem. Commun. 2009, (10), 1192-1194.

18. Blunt, M.; Lin, X.; Gimenez-Lopez, M. d. C.; Schroder, M.; Champness, N. R.; Beton, P. H. Directing Two-Dimensional Molecular Crystallization Using Guest Templates. Chem. Commun. 2008, (20), 2304-2306. 
19. Ciesielski, A.; Palma, C.-A.; Bonini, M.; Samorì, P. Towards Supramolecular Engineering of Functional Nanomaterials: Pre-Programming Multi-Component 2d SelfAssembly at Solid-Liquid Interfaces. Adv. Mater. 2010, 22, 3506-3520.

20. Nath, K. G.; Ivasenko, O.; Miwa, J. A.; Dang, H.; Wuest, J. D.; Nanci, A.; Perepichka, D. F.; Rosei, F. Rational Modulation of the Periodicity in Linear Hydrogen-Bonded Assemblies of Trimesic Acid on Surfaces. J. Am. Chem. Soc. 2006, 128, 4212-4213.

21. MacLeod, J. M.; Ivasenko, O.; Perepichka, D. F.; Rosei, F. Stabilization of Exotic Minority Phases in a Multicomponent Self-Assembled Molecular Network. Nanotechnology 2007, 18, 424031.

22. Kampschulte, L.; Werblowsky, T. L.; Kishore, R. S. K.; Schmittel, M.; Heckl, W. M.; Lackinger, M. Thermodynamical Equilibrium of Binary Supramolecular Networks at the Liquid-Solid Interface. J. Am. Chem. Soc. 2008, 130, 8502-8507.

23. Kampschulte, L.; Griessl, S.; Heckl, W. M.; Lackinger, M. Mediated Coadsorption at the Liquid-Solid Interface: Stabilization through Hydrogen Bonds. J. Phys. Chem. B 2005, 109, 14074-14078.

24. Li, M.; Xie, P.; Deng, K.; Yang, Y.-L.; Lei, S.-B.; Wei, Z.-Q.; Zeng, Q.-D.; Wang, C. A Dynamic Study of the Structural Change in the Binary Network in Response to Guest Inclusion. Phys. Chem. Chem. Phys. 2014, 16, 8778-8782.

25. Martsinovich, N.; Troisi, A. Modeling the Self-Assembly of Benzenedicarboxylic Acids Using Monte Carlo and Molecular Dynamics Simulations. J. Phys. Chem. C 2010, 114, $4376-4388$.

26. Florio, G. M.; Stiso, K. A.; Campanelli, J. S. Surface Patterning of Benzenecarboxylic Acids: Influence of Structure, Solvent, and Concentration on Molecular Self-Assembly. J. Phys. Chem. C 2012, 116, 18160-18174.

27. Heiko Schirmer, J. P., Jose Luis Martin, Juan R. Harto, Jose Carretero, Ulrich Niedballa Method for Producing Triiodine Trimesine Acid. WO2005005362A1, 2005. 
28. Sun, Y.; Bai, F. Y.; Min Wang, X.; Wang, Y.; Xian Sun, L.; Heng Xing, Y. A New Stable Porous Pr-Organic Framework Constructed by Multi-Iodine-Substituted Aromatic Polycarboxylates: Synthesis, Characterization, and Selective Adsorption of Dyes. Journal of Coordination Chemistry 2019, 72, 1560-1578.

29. Liu, C.-H.; Guan, Q.-L.; Yang, X.-D.; Bai, F.-Y.; Sun, L.-X.; Xing, Y.-H. PolyiodineModified 1,3,5-Benzenetricarboxylic Acid Framework Zn(Ii)/Cd(Ii) Complexes as Highly Selective Fluorescence Sensors for Thiamine Hydrochloride, Nacs, and Fe3+/Zn2+. Inorg. Chem. 2020, 59, 8081-8098.

30. Kresse, G.; Furthmüller, J. Efficient Iterative Schemes for Ab Initio Total-Energy Calculations Using a Plane-Wave Basis Set. Phys. Rev. B 1996, 54, 11169-11186.

31. Perdew, J. P.; Burke, K.; Ernzerhof, M. Generalized Gradient Approximation Made Simple. Phys. Rev. Lett. 1996, 77, 3865-3868.

32. Grimme, S. Semiempirical Gga-Type Density Functional Constructed with a LongRange Dispersion Correction. J. Comput. Chem. 2006, 27, 1787-1799.

33. https://www.3ds.com/products-services/biovia/products/molecular-modelingsimulation/biovia-materials-studio/.

34. Sun, H.; Mumby, S. J.; Maple, J. R.; Hagler, A. T. An Ab Initio Cff93 All-Atom Force Field for Polycarbonates. J. Am. Chem. Soc. 1994, 116, 2978-2987.

35. Nosé, S. A Unified Formulation of the Constant Temperature Molecular Dynamics Methods. J. Chem. Phys. 1984, 81, 511-519.

36. Parrinello, M.; Rahman, A. Polymorphic Transitions in Single Crystals: A New Molecular Dynamics Method. J. Appl. Phys. 1981, 52, 7182-7190.

37. Frisch, M. J.; Trucks, G. W.; Schlegel, H. B.; Scuseria, G. E.; Robb, M. A.; Cheeseman, J. R.; Scalmani, G.; Barone, V.; Petersson, G. A.; Nakatsuji, H., et al. Gaussian 16 Rev. B.01, Wallingford, CT, 2016. 
38. Chiodo, S.; Russo, N.; Sicilia, E. Lanl2dz Basis Sets Recontracted in the Framework of Density Functional Theory. J. Chem. Phys. 2006, 125, 104107.

39. Marenich, A. V.; Jerome, S. V.; Cramer, C. J.; Truhlar, D. G. Charge Model 5: An Extension of Hirshfeld Population Analysis for the Accurate Description of Molecular Interactions in Gaseous and Condensed Phases. Journal of Chemical Theory and Computation 2012, 8, 527-541.

40. Peyrot, D.; Silly, F. On-Surface Synthesis of Two-Dimensional Covalent Organic Structures Versus Halogen-Bonded Self-Assembly: Competing Formation of Organic Nanoarchitectures. ACS Nano 2016, 10, 5490-5498.

41. Claypool, C. L.; Faglioni, F.; Goddard, W. A.; Gray, H. B.; Lewis, N. S.; Marcus, R. A. Source of Image Contrast in Stm Images of Functionalized Alkanes on Graphite: A Systematic Functional Group Approach. J. Phys. Chem. B 1997, 101, 5978-5995.

42. Cyr, D. M.; Venkataraman, B.; Flynn, G. W.; Black, A.; Whitesides, G. M. Functional Group Identification in Scanning Tunneling Microscopy of Molecular Adsorbates. The Journal of Physical Chemistry 1996, 100, 13747-13759.

43. Ha, N. T. N.; Gopakumar, T. G.; Hietschold, M. Polymorphs of Trimesic Acid Controlled by Solvent Polarity and Concentration of Solute at Solid-Liquid Interface. Surf. Sci. 2013, 607, 68-73.

44. Mali, K. S.; Van Averbeke, B.; Bhinde, T.; Brewer, A. Y.; Arnold, T.; Lazzaroni, R.; Clarke, S. M.; De Feyter, S. To Mix or Not to Mix: 2d Crystallization and Mixing Behavior of Saturated and Unsaturated Aliphatic Primary Amides. ACS Nano. 2011, 5, 9122-9137. 
TOC graphic

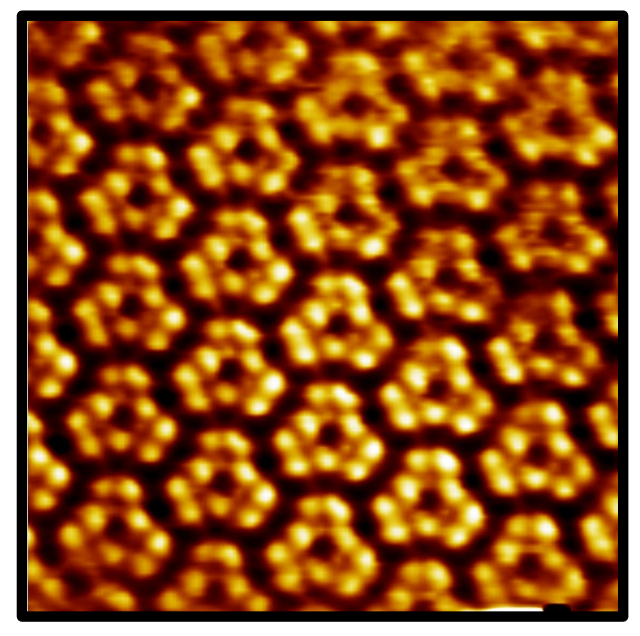

